

\title{
Lipase-Catalyzed Ring-Opening Polymerization of Benzyl Malolactonate: An Unusual Mechanism?
}

Hubert Casajus, Eric E. Dubreucq, Sylvain Tranchimand, Véronique Perrier, Caroline Nugier-Chauvin, Sandrine Cammas-Marion

\section{- To cite this version:}

Hubert Casajus, Eric E. Dubreucq, Sylvain Tranchimand, Véronique Perrier, Caroline Nugier-Chauvin, et al.. Lipase-Catalyzed Ring-Opening Polymerization of Benzyl Malolactonate: An Unusual Mechanism?. Biomacromolecules, 2020, 21 (7), pp.2874-2883. 10.1021/acs.biomac.0c00593 . hal-02890150

\section{HAL Id: hal-02890150 \\ https://hal.science/hal-02890150}

Submitted on 9 Jul 2020

HAL is a multi-disciplinary open access archive for the deposit and dissemination of scientific research documents, whether they are published or not. The documents may come from teaching and research institutions in France or abroad, or from public or private research centers.
L'archive ouverte pluridisciplinaire HAL, est destinée au dépôt et à la diffusion de documents scientifiques de niveau recherche, publiés ou non, émanant des établissements d'enseignement et de recherche français ou étrangers, des laboratoires publics ou privés. 


\section{Lipase catalyzed ring-opening polymerization of benzyl malolactonate: an unusual mechanism?}

Hubert Casajus ${ }^{1, \dagger}$, Eric Dubreucq ${ }^{2}$, Sylvain Tranchimand ${ }^{1}$, Véronique Perrier ${ }^{2}$, Caroline NugierChauvin $^{1}$, Sandrine Cammas-Marion ${ }^{1,3 *}$

1. Univ Rennes, ENSCR, CNRS, ISCR (Institut des Sciences Chimiques de Rennes) - UMR 6226, F-35000 Rennes, France.

2. L'Institut Agro - Montpellier SupAgro, Univ Montpellier, INRA, CIRAD - UMR IATE, F-34060 Montpellier, France

3. Univ Rennes, INSERM, INRA, Institut NUMECAN (Nutrition Metabolisms and Cancer) UMR_A 1341, UMR_S 1241, F-35000 Rennes, France.

KEYWORDS: Lipase-Catalyzed Polymerization, Ring-Opening Polymerization, Mechanism, Benzyl Malolactonate, CpLip2.

ABSTRACT: The use of safe natural catalyst such as enzymes for ring opening polymerization (ROP) of $\beta$-substituted $\beta$-lactones such as benzyl malolactonate (MLABe) is an important objective considering the biomedical applications of the resulting (co)polymers. However, the preparation of well-defined polymeric materials using such systems requires an understanding of enzymesubstrate interactions. In this context, we investigated the mechanism of lipase-catalyzed ROP of MLABe, because it appears that it is probably not the same as the one widely described for other lactones such $\varepsilon$-caprolactone, propiolactone and lactide. Enzymatic-catalyzed ROPs of MLABe in presence of the lipase/acyltransferase CpLip2 and its serine knock-out (serine KO) mutant (CpLip2_180A) have led to poly(benzyl malate) (PMLABe) terminated by a monobenzyl fumarate group with monomer conversion higher than $70 \%$ and weight average molar mass of about 3,600 $\mathrm{g} / \mathrm{mol}(=1.42)$. On the other hand, only less than $7 \%$ of MLABe conversion and no polymer formation were observed when the polymerization reaction was conducted in presence of inactivated CpLip2 (heated at $100^{\circ} \mathrm{C}$ ). Moreover, the ROP of MLABe in presence of imidazole, a synthetic mimic of the catalytic histidine, led to a PMLABe terminated by a monobenzyl fumarate group. On the contrary, neither the enzymatic-catalyzed ROP of benzyl dimethylmalolactonate (diMeMLABe), a MLABe with two methyl groups instead of the two "acidic" protons on the lactone's ring, in presence of CpLip2 and CpLip2_180A nor its chemical ROP in presence of imidazole were successful. Together, all these results suggested that the lipase-catalyzed polymerization of malolactonates occurred through the abstraction of one of the two "acidic" protons of the lactone's ring by the histidine of the catalytic triad leading to the corresponding monobenzyl fumarate responsible for the polymerization of the remaining monomer. Finally, molecular modeling of the positioning of the monomer into the catalytic site of the CpLip2 and DFT quantum-chemical calculations highlighted an interaction of (R) and (S)-MLABe with the 
catalytic histidine of the enzyme preferentially to serine, in the form of a strong hydrogen bond with one of the "acidic" protons of MLABe, thus supporting the important role of the catalytic histidine in the polymerization of such cyclic lactones.

INTRODUCTION. Aliphatic polyesters belong to a polymer family with outstanding biocompatibility and biodegradability properties particularly appreciated when such polymeric materials are designed for applications in the biomedical field. Such polymers, being in contact with human bodies, must follow very strict specifications: they must be non-toxic, nonimmunogenic, non-carcinogenic and non-thrombogenic ${ }^{1}$. Moreover, their biodegradation must lead to low molar mass molecules, which are respectful of the previously cited specifications and/or eliminated/excreted from the body. These polyesters are usually synthesized by polymerization reactions using chemical catalysts through either the direct polycondensation of a diacid with a diol, for example, or ring opening polymerization (ROP) of lactones or lactide ${ }^{2}$. The ROP technique allows the obtention of polymers having high and controlled molar masses as well as defined structures ${ }^{3}$. On the other hand, the ROP reactions in presence of cationic or anionic initiators often requires the addition of organometallic catalysts whose presence even as traces might lead to unwanted toxicity ${ }^{4}$.

Consequently, alternative synthetic methods are developed to overcome this major drawback, more particularly when such (co)polymers are synthesized for human health applications. Therefore, the use of biological catalysts such as lipases is of growing interest. Indeed, enzymes allow polymerization reactions under mild conditions (temperature, $\mathrm{pH}$ and pressures), avoid the use of organic solvents and most importantly are generally not toxic ${ }^{5}$. The synthesis of polyesters through ROP of their corresponding cyclic monomers in the presence of lipases was described for the first time in 1993 by Kobayashi et al. ${ }^{6}$ and Kohn et al. ${ }^{7}$, who synthesized a poly( $\varepsilon$-caprolactone) thanks to the ROP of $\varepsilon$-caprolactone in the presence of Pseudomonas fluorescens lipase and porcine pancreatic lipase (PPL), respectively, as catalysts. These preliminary results have opened the way towards enzymatic-catalyzed polymerization reactions leading to "greener" and more biocompatible polyesters such as poly( $\varepsilon$-caprolactone $)^{8,9}$.

In this context, we have evaluated the possibilities of using lipase-based ROP of a special family of lactones, i.e. the $\beta$-substituted $\beta$-lactones also called alkyl malolactonates, to synthesize poly(malic acid) and its derivatives ${ }^{10,11}$. In a first step, we have established, through a Design of Experiment (DoE) procedure, the optimal experimental conditions to reproducibly synthesize poly(benzyl malate) with weight average molar masses varying from 5,000 to 13,000 g/mol, dispersities between 1.2 and 1.6 (depending on the conditions used for the polymerization) and hydrolysis rates lower than 5\%, using PPL as catalyst ${ }^{10}$. PMLABe obtained through lipasecatalyzed ROP of MLABe were then formulated and the resulting nanoparticles were involved in several in vitro assays whose results have highlighted the influence of the polymer synthesis method on cell viability in particular ${ }^{11}$. Moreover, physico-chemical characteristics of PMLABe obtained through lipase-catalyzed ROP of racemic and optically active MLABe led us to question the mechanism of this enzymatic polymerization ${ }^{11}$. Indeed, we have previously shown that the 
lipase-catalyzed ROP of R-MLABe and S-MLABe occurred through an O-alkyl bound cleavage with an inversion of configuration of the asymmetric carbon of the lactone's ring, as in the case of anionic ROP of MLABe for which the initiator attacks on the asymmetric carbon of the lactone's ring leading to a ring opening through an O-alkyl bound cleavage and an inversion of configuration of the asymmetric carbon ${ }^{11}$. On the contrary, the mechanism described, and well-accepted, for the lipase-catalyzed ROP of unsubstituted and $\alpha, \beta$-alkyl substituted lactones ( $\varepsilon$-caprolactone, butyrolactone, lactide, etc.) involved an O-acyl bound cleavage as the result of the attack of the activated catalytic serine onto the carbonyl of the lactone and the formation of an enzymemonomer intermediate (canonical mechanism) $)^{12-18}$. Therefore, it seems that the lipase-catalyzed ROP of MLABe does not follow the canonical mechanism described for other lactones and lactides, but rather the one described for its anionic ROP ${ }^{11}$. Consequently, we have realized several experiments involving the use of the lipase/acyltransferase CpLip2 enzyme (a lipase with high affinity for nucleophiles other than water ${ }^{19}$ ), a modified CpLip2 which catalytic serine was mutated into an alanine residue (serine-KO), an inactivated CpLip2 obtained by heating at $100^{\circ} \mathrm{C}$, a MLABe derivative on which the two "acidic" protons of the lactone's ring have been changed by two methyl groups, synthetic mimics of the three amino acids of the lipase's catalytic triad and several molecular modeling experiments as well as DFT quantum-chemical calculations. The obtained results described in the present paper allowed us to propose a mechanism for the lipase-catalyzed ROP of malolactonates.

MATERIALS AND METHODS. All chemicals were used as received. Porcine pancreatic lipase (PPL) was purchased from Sigma-Aldrich Co. Dimethyl benzyl malolactonate (diMeMLABe) was a gift from Kymia Nova (Saclay, France). Benzyl malolactonate (MLABe) was synthesized according to literature ${ }^{20}$.

Nuclear Magnetic Resonance spectroscopy: The standard temperature was adjusted to 298K. NMR spectra were recorded on a Bruker Avance III 400 spectrometer operating at $400.13 \mathrm{MHz}$ for ${ }^{1} \mathrm{H}$, equipped with a BBFO probe with a Z-gradient coil and a GREAT 1/10 gradient unit. The zg30 Bruker pulse program was used for $1 \mathrm{D}^{1} \mathrm{H}$ NMR, with a TD of $64 \mathrm{k}$, a relaxation delay $\mathrm{d} 1=$ $2 \mathrm{~s}$ and 8 scans. The spectrum width was set to $18 \mathrm{ppm}$. Fourier transform of the acquired FID was performed without any apodization in most cases.

Size Exclusion Chromatography (SEC): Weight average molar mass $\left(\mathrm{M}_{\mathrm{w}}\right)$ and dispersity $(\bigoplus=$ $\mathrm{M}_{\mathrm{w}} / \mathrm{M}_{\mathrm{n}}$ ) values were measured by SEC in THF at $40{ }^{\circ} \mathrm{C}$ (flow rate $=1.0 \mathrm{~mL} / \mathrm{min}$ ) on a GPC2502 Viscotek apparatus equipped with a refractive index detector Viscotek VE 3580 RI, a guard column Viscotek TGuard, Org 10x4.6mm, a LT5000L gel column (for samples soluble in organic medium) 300 x $7.8 \mathrm{~mm}$ and a GPC/SEC OmniSEC Software. The polymer samples were dissolved in THF ( $2 \mathrm{mg} / \mathrm{mL}$ ). All elution curves were calibrated with polystyrene standards (weight average molar masses: 1,$240 ; 2,630 ; 6,220 ; 13,700 ; 30,100$ and 78,200 g/mol).

Preparation of the CpLip2 and CpLip2_S180A (serine KO) lipases: Recombinant lipase/acyltransferase CpLip2 from Candida parapsilosis CBS 604 was produced by heterologous expression in Komagataella phaffii (formerly named Pichia pastoris) as described previously ${ }^{21}$. 
The serine KO mutant CpLip2_S180A was obtained by site-directed mutagenesis of serine S180 into an alanine residue. The mutation was performed by Life Technologies (Regensburg, Germany) on the gene of CpLip2 in fusion with the signal peptide of the alpha mating factor of Saccharomyces cerevisiae within the pPICZ $\alpha \mathrm{B}$ plasmid, that was used to transform K. phaffii according to the work already described elswhere ${ }^{22}$. The protein was produced in a $1 \mathrm{~L}$ bioreactor and purified as described earlier ${ }^{22}$.

Enzymatic polymerization of MLABe: The different PMLABe were synthesized as described previously [10] by ring-opening polymerization of the MLABe in presence of the enzyme (PPL, CpLip2 or CpLip2_S180A) in Tris-HCl buffer pH7. Experiments were realized in a 24-multi reactor Büchi Syncore Line. In a $30 \mathrm{~mL}$-tube, enzyme (8.4 mg) was mixed with pure MLABe (336 mg) and Tris-HCl buffer (125 $\mu \mathrm{L}, \mathrm{pH} 7,105 \mathrm{mM})$. The mixture was stirred at $390 \mathrm{rpm}$ and $60{ }^{\circ} \mathrm{C}$. After $72 \mathrm{~h}$, reaction was stopped by the addition of $3 \mathrm{~mL}$ of THF and $3 \mathrm{~mL}$ of chloroform. The mixture was then transferred into a separating funnel. Separations were realized by adding $15 \mathrm{~mL}$ of distilled water and $15 \mathrm{~mL}$ of chloroform. Organic phases were dried over $\mathrm{MgSO}_{4}$ and filtered. Finally, solvents were evaporated under reduced pressure. The crude polymers were analyzed by ${ }^{1} \mathrm{H}$ NMR to determine monomer's conversion (from 70 to $90 \%$ ) and hydrolysis rate (below 3\%). Then, they were dissolved in chloroform and precipitated in a large excess of cold diethyl ether to eliminate unreacted monomer and impurities with a recovering yield of 70 to $90 \%$. After elimination of the supernatant, polymers were dried under vacuum and analyzed by ${ }^{1} \mathrm{H}$ NMR and SEC.

\section{Polymerization of MLABe in presence of the synthetic catalytic triad:}

- Synthesis of imidazolium trifluoroacetate salt ${ }^{23}$ : In a round-bottomed flask, $505 \mathrm{mg}$ (7.42 mmol, 1 eq.) of imidazole were dissolved in $50 \mathrm{~mL}$ of chloroform. This solution was cooled to $0^{\circ} \mathrm{C}$, then, $1.25 \mathrm{~mL}$ (16.32 mmol, 2.2 eq.) of trifluoracetic acid (TFA) was added dropwise. After $24 \mathrm{~h}$ stirring, the precipitated salt was filtered, washed with chloroform and dried overnight at $80^{\circ} \mathrm{C} .578 \mathrm{mg}$ of imidazolium salt were obtained (yield $=43 \%$ ). Melting point: $\mathrm{P}_{\mathrm{m}}$, ref $=127.2^{\circ} \mathrm{C}$ [20], $\mathrm{P}_{\mathrm{m} \text {, measured }}=128{ }^{\circ} \mathrm{C}$.

- Polymerization reactions: Experiments were realized in a 24-multi reactor Büchi Syncore Line. In a $30 \mathrm{~mL}$-tube, imidazolium salt and/or imidazole, and/or TFA, and/or benzyl alcohol (composition depending of the entry as shown by data gathered in Table 1) were mixed with MLABe (200 mg, $0.97 \mathrm{mmol}$, 1eq.).

Table 1. Composition of the reactive mixture.

\begin{tabular}{|c|c|c|c|c|}
\hline Entry & Imidazolium salt & Benzyl alcohol & TFA & Imidazole \\
\hline 1 & $35.3 \mathrm{mg} / 0.194 \mathrm{mmol} / 0.2 \mathrm{eq}$. & $4 \mu \mathrm{L} / 0.039 \mathrm{mmol} / 0.04 \mathrm{eq}$. & 0 & 0 \\
\hline 2 & $35.3 \mathrm{mg} / 0.194 \mathrm{mmol} / 0.2 \mathrm{eq}$ & 0 & 0 & 0 \\
\hline 3 & 0 & $4 \mu \mathrm{L} / 0.039 \mathrm{mmol} / 0.04 \mathrm{eq}$. & 0 & 0 \\
\hline 4 & 0 & 0 & $14.8 \mu \mathrm{L} / 0.194 \mathrm{mmol} / 0.2 \mathrm{eq}$. & $13.2 \mathrm{mg} / 0.194 \mathrm{mmol} / 0.2 \mathrm{eq}$. \\
\hline 5 & 0 & 0 & 0 & $113 \mu \mathrm{L} / 0.173 \mu \mathrm{mol} / 1.07^{*} 10^{-4} \mathrm{eq}$. \\
\hline 6 & 0 & 0 & 0 &
\end{tabular}


In parallel, ROP of MLABe $\left(\mathrm{m}=331 \mathrm{mg}, 1.6 * 10^{-3} \mathrm{~mol}\right)$ in presence of imidazole $\left(\mathrm{n}=1.73^{*} 10^{-7}\right.$ mol, $113 \mu \mathrm{L}$ of a stock solution at a concentration of $0.1 \mathrm{~g} / \mathrm{L}$ ) in a catalytic amount, i.e. the same imidazole mole number than the one corresponding to $8.3 \mathrm{mg}$ of PPL (Molar mass $=48,000 \mathrm{~g} / \mathrm{mol}$, $\mathrm{n}=1.73 * 10^{-7} \mathrm{~mol}$ ), was conducted in $172 \mu \mathrm{L}$ of Tris-HCl buffer at $\mathrm{pH} 7$. After $72 \mathrm{~h}$ at $60{ }^{\circ} \mathrm{C}$ and $390 \mathrm{rpm}$, reactions were stopped by the adding $5 \mathrm{~mL}$ of chloroform. The mixture was then filtered and transferred in a separating funnel. Separation was realized by adding $15 \mathrm{~mL}$ of distilled water and $15 \mathrm{~mL}$ of chloroform. The aqueous phase was extracted twice by $7 \mathrm{~mL}$ of chloroform. Organic phases were dried over $\mathrm{MgSO}_{4}$ and filtered. Finally, chloroform was evaporated under vacuum. The crude polymers were analyzed by ${ }^{1} \mathrm{H}$ NMR to determine monomer's conversion and hydrolysis rate. Then, they were dissolved in chloroform and precipitated in a large excess of cold diethyl ether. After elimination of the supernatant, polymers were dried under vacuum at room temperature, and characterized by SEC and ${ }^{1} \mathrm{H}$ NMR.

Computational methods: The 3D structure of CpLip2 lipase/acyltransferase ${ }^{24}$ was refined by molecular dynamics and energy minimization in an explicit TIP3P water box as described earlier ${ }^{25-}$ 27. The initial geometry of R- and S-MLABe was determined by energy minimization using the density functional tight binding (DFTB) quantum chemical method with the GFN1-xTB model ${ }^{28}$ and the $\mathrm{COSMO}^{29}$ water solvation model implemented in the DFTB module of AMS 2019 (SCM, Amsterdam, NL). Molecular docking was performed with Autodock Vina v1.1.2 ${ }^{30}$ using the UCSF Chimera v1.14.1 ${ }^{31}$ interface. The energy of the structures of the two best poses was minimized within Chimera using the AMBER ff14SB and the AM1-SCC force fields for atomic partial charge calculations in the protein and the ligand, respectively, with the protein backbone constrained to fixed positions, allowing the selection of the conformation with the lowest estimated energy. For each ligand, sub-models restricted to amino acid residues with at least one atom closer than $5 \AA$ from the ligand (475-480 atoms) were constructed. The broken amide bonds of the protein backbone were restored by capping with $-\mathrm{CO}-\mathrm{CH}_{3}$ and $-\mathrm{NH}-\mathrm{CH}_{3}$ groups on the amino and carboxyl sides, respectively, in line with the backbone of the former neighboring residues. The geometry of the sub-models was then refined using DFTB (GFN1-xTB and COSMO water implicit solvation models) for energy minimization, with the position of $\mathrm{C}$ and $\mathrm{N}$ atoms of the protein backbone constrained to fixed positions. A final optimization used DFT for energy minimization, with MLABe and all the atoms (and bound hydrogen atoms) closer than $3 \AA$ from the ligand being free to move and the other atoms being fixed as a bloc. DFT calculations were performed using the BP86 GGA functional with D4(EEQ) dispersion correction ${ }^{32}$, the DZ double $\zeta$ basis set with a large frozen core and the COSMO water solvation model within the ADF module of AMS. The interactions between MLABe and the protein sub-model, both defined as closed-shell molecular fragments, were then analyzed at the same level of DFT by the extended transition state-natural orbitals for chemical valence (ETS-NOCV) energy decomposition method ${ }^{33}$ using ADF. Graphical representations were obtained with UCSF Chimera v1.14 ${ }^{31}$.

RESULTS AND DISCUSSION. Lipases are enzymes belonging to the family of hydrolases, class 3 in the EC nomenclature (Enzyme Commission numbers). More specifically, they are serine 
$\alpha / \beta$ hydrolases which have the particularity of being active in presence of an interface between an aqueous phase (water or buffer) and an organic phase (hydrophobic substrate and/or organic solvent). Present in plants, insects, animals and microorganisms, lipases play a key role in the degradation and synthesis of lipids, and catalyze, under physiological conditions, the hydrolysis of mono-, di- and triglycerides ${ }^{34}$. The Protein Data Bank (PDB) records more than 100 threedimensional structures of lipases highlighting the following highly conserved structural elements specific to these enzymes $^{35}$ : i). The $\alpha / \beta$ folding composed by eight $\beta$ sheets and six $\alpha$ helices; ii). The catalytic triad composed by a nucleophilic serine, an aspartate/glutamate and a histidine; iii). The oxyanion hole formed by two active amino acids (or more depending on the lipase) of the active site, stabilizing the enzyme/substrate reaction intermediate through hydrogen bonds between the substrate and the nitrogen of the amide function; iv). The lid formed by one or more $\alpha$ helices which is opened in presence of the activation interface thus allowing the positioning of the substrate into the active site; $v$ ). The active site, mostly composed of hydrophobic amino acids, and varying from enzyme to another thus explaining the difference in specificity observed in lipases $^{35}$. The mechanism of ester functions hydrolysis, called canonical mechanism, involves the three amino acids (aspartate, histidine and serine) of the catalytic triad: briefly, the serine, activated by the aspartate and histidine, forms a covalent acyl-enzyme intermediate (acylation step) which is then hydrolyzed thanks to the attack of water molecules activated by the histidine (deacylation step $)^{35}$. The structure of the acyl-enzyme intermediate has been observed for a deacylation step slower than the acylation one and, together with detailed kinetic studies, validated and confirmed the canonical mechanism ${ }^{35}$.

Lipase-catalyzed ROP mechanism of unsubstituted lactones (such as $\varepsilon$-caprolactone, lactide) and $\alpha / \beta$-alkyl substituted $\beta$-lactones (such as butyrolactone, $\alpha$-methyl propiolactone) has been already described in the literature ${ }^{12-18}$. It uses the canonical mechanism as a basis, and is widely accepted by the entire scientific community (Scheme 1). In a first step, the lactone ring is opened by the lipase (Scheme 1.1): the activated serine attacks the carbonyl of the lactone, thus forming a tetrahedral intermediate, and the reformation of the carbon-oxygen double bond leads to the opening of the lactone and to the formation of an acyl-enzyme intermediate. Then, three possibilities exist for the de-acylation of the enzyme (Scheme 1.2): i). Initiation: the initiator (here water) attacks the enzyme-substrate complex leading to the formation of an activated open monomer. Conversely, the formation of an enzyme-substrate complex can be observed when the terminal acid function of the growing monomer or polymer reacts with catalytic serine. ii). Propagation: a growing monomer or polymer attacks the enzyme-substrate complex leading to the growth of the macromolecular chain. Conversely, lipase can randomly hydrolyze an ester function of the polymer, thereby forming two polymers with smaller degrees of polymerization. iii). Cyclization: the terminal alcohol function of the polymer reacts with the ester function between the lipase to the polymer. Conversely, lipase can hydrolyze an ester function of a cyclic polymer and thus leading to the corresponding linear polymer. 

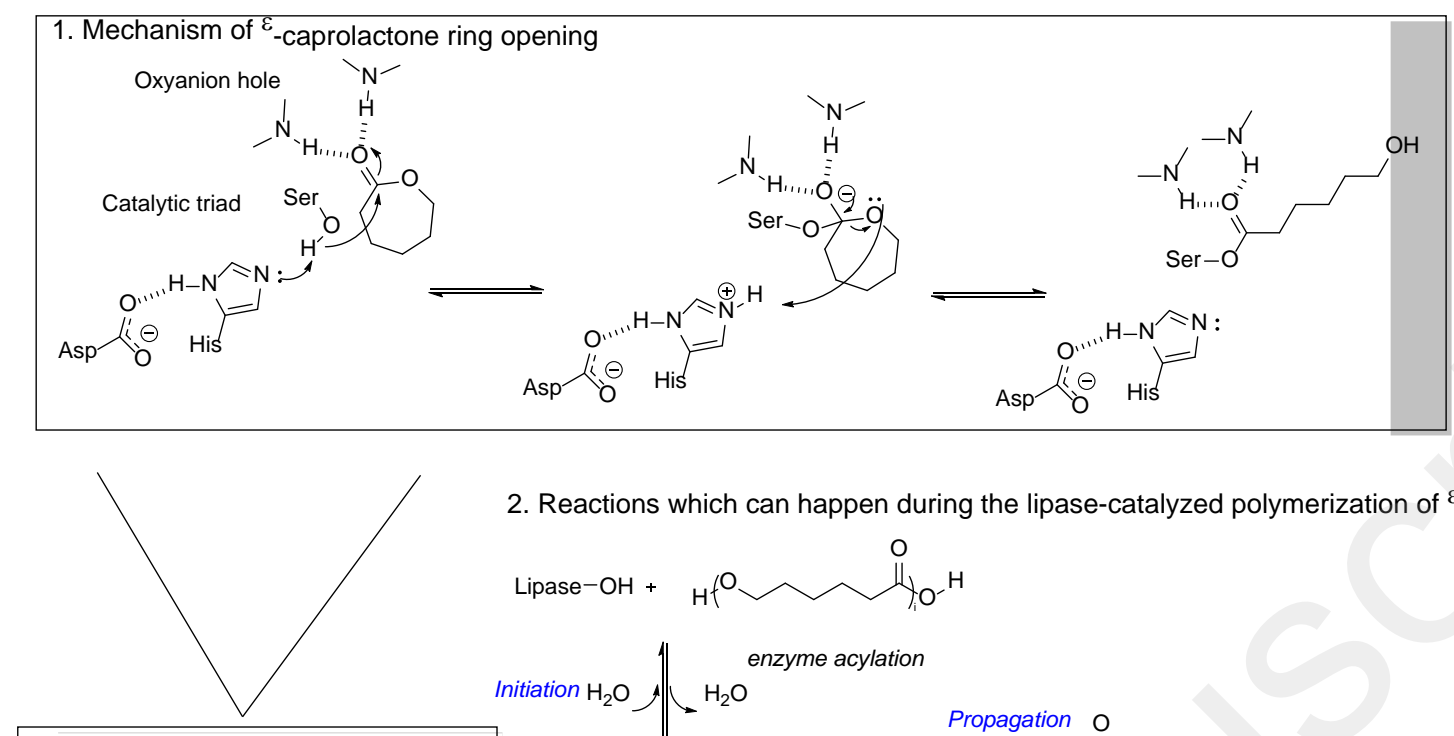

2. Reactions which can happen during the lipase-catalyzed polymerization of ${ }^{\varepsilon}$-caprolactone Lipase- $\mathrm{OH}+\mathrm{H}^{\mathrm{O}} \mathrm{H}_{\mathrm{i} \mathrm{O}_{-}} \mathrm{H}$

Initiation $\mathrm{H}_{2} \mathrm{O}$

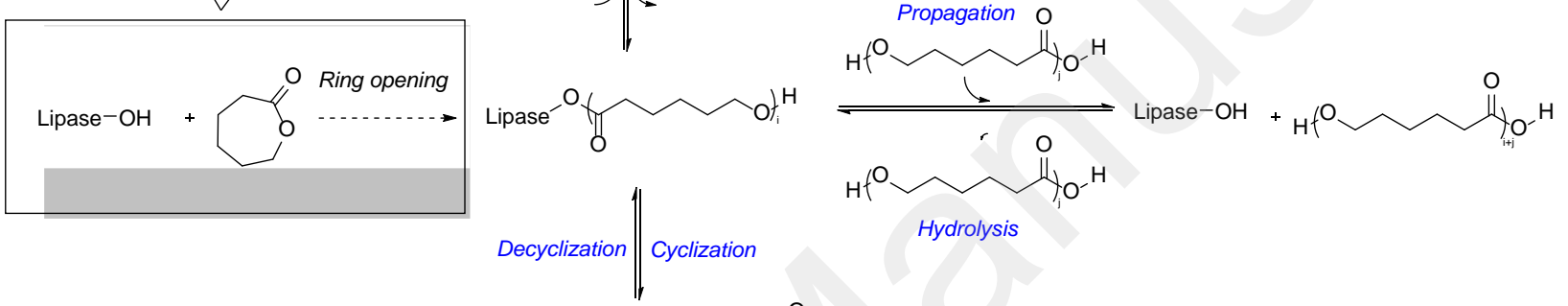

Lipase-OH

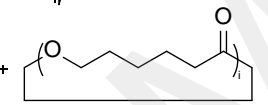

Scheme 1. Canonical mechanism of lipase-catalyzed ROP of unsubstituted and $\alpha / \beta$-alkyl substituted $\beta$-lactones ${ }^{5,12}$.

On the other hand, and contrary to the other lactones mentioned above, the mechanism of enzymatic polymerization of alkyl malolactonates is still not elucidated. The question "Does the lipase-catalyzed ROP of alkyl malolactonate follow the same mechanism as the one described for chemical ROP of alkyl malolactonate (Scheme 2) or the one described by several authors for unsubstituted lactones (Scheme 1)?” is still unanswered right now.

<smiles>[R]C(=O)OC(CC(=O)O)C(=O)O</smiles><smiles>[R]OC(=O)C1CC(=O)O1</smiles><smiles>[R]OC(=O)C(C)OC(=O)CC(C)(CC(=O)O[3H])C(=O)O[R]</smiles>

Scheme 2. Mechanism of the chemical ROP of alkyl malolactonate ${ }^{20}$.

The mechanism described in Scheme 1 for the lipase-catalyzed ROP of unsubstituted lactones and lactide is based on a nucleophilic attack of the catalytic serine on the carbonyl of the monomer, leading to the ring opening through an O-acyl bound cleavage. Concerning the lipase-catalyzed ROP of benzyl malolactonate (MLABe), we have recently demonstrated that the lactone ring 
opening might occur through an O-alkyl bound cleavage, as for the anionic ROP of MLABe for which the initiator (tetraethylammonium benzoate or other carboxylate salts) attacks on the asymmetric carbon of the alkyl malolactonate thus leading to an O-alkyl ring opening ${ }^{11,20}$. Indeed, the polymers synthesized from R-MLABe using either PPL as catalyst or tetraethylammonium benzoate as initiator have identical rotational powers with the same sign whatever the route of synthesis ${ }^{11}$. If this result seems logical in view of the alkyl malolactonate reactivity, it is much less if the reaction mechanism is considered as a whole. Indeed, according to literature and canonical mechanism, the catalytic serine should react with the carbonyl group of the monomer ring to form an acyl-enzyme intermediate through a hydrolyzable ester bond ${ }^{12-18}$. In the case of lipase catalyzed ROP of alkyl malolactonate, if the attack of the catalytic serine on the asymmetric carbon of the lactone is considered, an alkyl-enzyme intermediate through hardly hydrolyzable ether bound should be obtained. However, we never observed such intermediate in ${ }^{1} \mathrm{H}$ NMR spectra and SEC chromatograms of both the crude and purified products. Therefore, it would seem that the canonical mechanism cannot be applied in the case of lipase-catalyzed ROP of alkyl malolactonate. In an attempt to understand the mechanism involved in the lipase-catalyzed ROP of this unusual lactones' family, several experiments were conducted. Coulembier et al. ${ }^{23}$ designed and used a synthetic catalytic triad to study lipase-catalyzed polymerization of D- and L-lactide. As shown by Figure 1, this synthetic catalytic triad is composed by trifluoroacetate mimicking the aspartate with however a lower nucleophilic character, imidazole for the histidine and benzyl alcohol mimicking the catalytic serine.

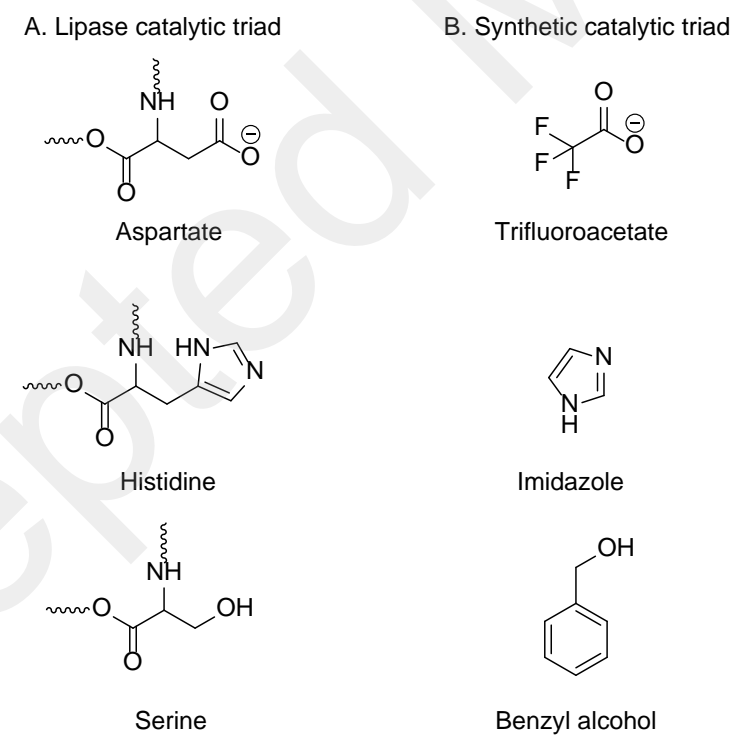

Figure 1. The lipase (A) and synthetic (B) catalytic triad.

Therefore, such synthetic catalytic triad was synthesized and tested for the polymerization of MLABe. The reaction between the MLABe and the synthetic catalytic triad, complete or partial, was carried out at $60{ }^{\circ} \mathrm{C}$ and $390 \mathrm{rpm}$ (conditions similar to those of the enzymatic polymerization). Six polymerization conditions were tested as summarized in Table 1 ("Materials and Methods”). 
The crude PMLABe were analyzed by proton NMR (conversion rate and hydrolysis rate) and purified PMLABe were analyzed by SEC (dispersity and weight average molar mass, Figure SI.1). As shown by the results gathered in Table 2, the complete synthetic catalytic triad (Table 2, Entry i) and the mixture trifluoroacetic acid/imidazole (Table 2, Entry ii) are able to polymerize MLABe, but the weight average molar masses of the resulting polymers are very low $(600 \mathrm{~g} / \mathrm{mol}$ and 500 $\mathrm{g} / \mathrm{mol}$ ). Moreover, no polymerization reaction was observed when MLABe was brought together with benzyl alcohol (Table 2, Entry iii) or trifluoroacetic acid (Table 2, Entry iv) alone. On the other hand, 0.2 equivalent of imidazole (Table 2, Entry $v$ ) and catalytic amount of imidazole equivalent to the one of histidine into the catalytic triad (Table 2, Entry vi) are able to effectively polymerize MLABe with $100 \%$ monomer conversion leading to PMLABe with quite high weight average molar masses and low dispersities (3,350 g/mol, dispersity of 1.50 for Entry $v$, and 7,370 $\mathrm{g} / \mathrm{mol}$, dispersity of 1.15 for Entry vi). It should be noted that no monomer's hydrolysis has been observed whatever the experimental conditions used.

Table 2. Characteristics of the PMLABe obtained by ROP of MLABe with the synthetic catalytic triad, complete or partial.

\begin{tabular}{|c|c|c|c|c|}
\hline Entry & Conditions & $\begin{array}{c}\text { Conversion } \\
\text { rate }^{\mathrm{a}}(\%)\end{array}$ & $\mathrm{M}_{\mathrm{w}}^{\mathrm{b}}(\mathrm{g} / \mathrm{mol})$ & $\bigoplus^{\mathrm{b}}$ \\
\hline (i) & Imidazolium salt (0.2 eq.) + benzyl alcohol (0.04 eq.) & 100 & 600 & 1.40 \\
\hline (ii) & Imidazolium salt (0.2 eq.) & 100 & 500 & 1.40 \\
\hline (iii) & Benzyl alcohol (0.04 eq.) & 0 & --- & --- \\
\hline (iv) & Trifluoroacetate (0.2 eq.) & 0 & --- & -- \\
\hline (v) & Imidazole (0.2 eq.) & 100 & 3,350 & 1.50 \\
\hline (vi) & Imidazole $\left(1.07 * 10^{-4}\right.$ eq. $)$ & 100 & 7,370 & 1.15 \\
\hline
\end{tabular}

a. Determined from ${ }^{1} \mathrm{H}$ NMR spectra of crude products; $b$. Measured by SEC (THF, Polystyrene standards, 1 $\mathrm{mL} / \mathrm{min}, 40^{\circ} \mathrm{C}$; Figure SI-1)

In this experiment, imidazole can be considered as the "synthetic" pendant of histidine of the lipase catalytic triad. From the results obtained, it seems logical to ask whether histidine alone is able to polymerize MLABe, without the intervention of serine and, therefore, of the canonical mechanism. An acid-base mechanism excluding the serine would therefore be possible. Indeed, this kind of mechanism has already been described for PPL-catalyzed aldolization reaction between an aldehyde and a ketone in the presence of water ${ }^{36}$. Moreover, O-alkyl ring-opening has been described for unsubstituted lactones when they are polymerized in the presence of a weak base as initiator: the carboxylate ion thus formed would then be the species responsible for the propagation ${ }^{37-39}$. However, the formation of a carboxylate in this case would come from side reactions, including deprotonations of the monomer or the growing polymer chains ${ }^{38}$. Indeed, Kricheldorf et al. have shown that anionic initiators, such as alcoholate, or basic initiators, such as tertiary amine, could abstract one of the two "acidic" protons of the lactone's ring in $\alpha$ position of the carbonyl to form the corresponding acrylate (Scheme 3). 

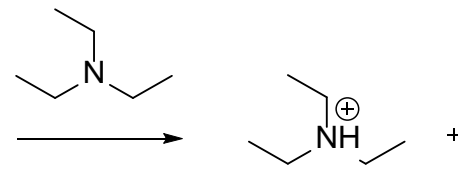

$\overbrace{0}{ }^{\Theta}$

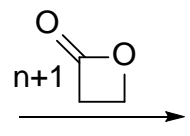<smiles>C=CC(=O)OCCC(=O)OCCC(=O)O[O-]</smiles>

Scheme 3. Mechanism of the propiolactone ROP in presence of a tertiary amine as initiator ${ }^{38}$.

This acrylate would continue the polymerization of the remaining monomers thus leading to a polymer terminated at one end by a double bond. Furthermore, Guérin et al. have shown that these deprotonation reactions also take place during the anionic ROP of alkyl malolactonates ${ }^{40}$.

If this deprotonation reaction is considered for the lipase-catalyzed polymerization of MLABe, the first step of the mechanism would consist in the abstraction of an "acidic" proton of the lactone's ring in $\alpha$ position of the carbonyl by the basic nitrogen group of imidazole (Figure 2A).

A. Benzyl malolactonate (MLABe)

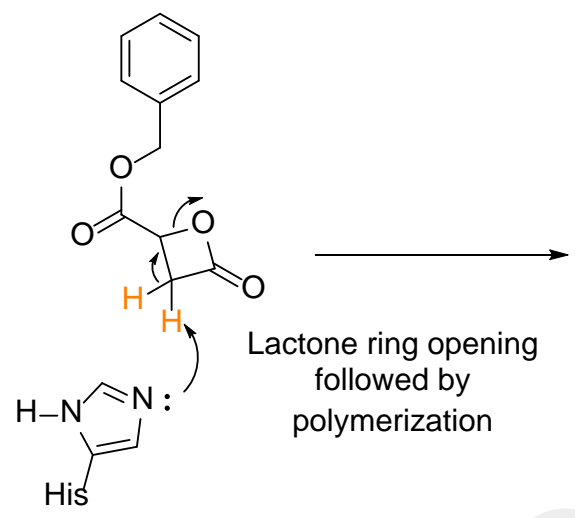

B. Benzyl dimethylmalolactonate (diMeMLABe)

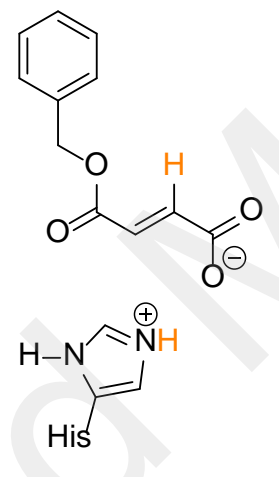

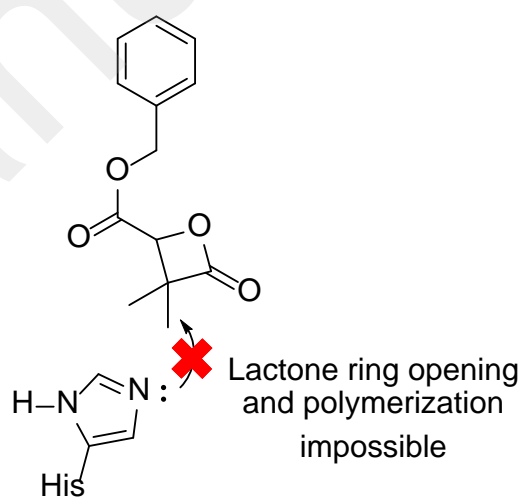

Figure 2. Proposed first step for the lipase-catalyzed ROP of: A. MLABe, and B. diMeMLABe.

The carboxylate thus formed would then continue the polymerization of the remaining MLABe. Therefore, if the two "acidic" protons of MLABe's ring are replaced by methyl groups, which is the case for dimethyl-MLABe, then this first step could no longer take place and the polymerization would not start (Figure 2B).

The enzymatic polymerization of benzyl dimethyl malolactonate (diMeMLABe) was therefore carried out using the optimized protocol established for the polymerization of MLABe. PPL was mixed with diMeMLABe (1/40 PPL/diMeMLABe ratio) in Tris-HCl buffer (105 mM, pH 7), and the mixture was stirred at $390 \mathrm{rpm}$ during $72 \mathrm{~h}$ at $60^{\circ} \mathrm{C}$. The obtained crude product was analyzed by proton NMR and the spectrum showed that no polymerization reaction took place. Indeed, no characteristic peak of a polymer was observed while all the peaks of diMeMLABe (Figure SI.2A) were present thus highlighting both the non-degradation and non-polymerization of this monomer by PPL (Figure SI.2B). 
The impossibility to polymerize the diMeMLABe using PPL as catalyst might be in favor of the proposed mechanism (abstraction of one of the two "acidic" protons from the lactone's ring by the catalytic histidine), even if we cannot ignore that this impossibility might be also due to steric hindrance brought by the presence of the methyl groups on the lactone ring.

Therefore, we carefully analyzed the proton NMR spectra of PMLABe obtained by PPL-catalyzed ROP of MLABe in Tris-HCl buffer pH7 (Figure 3).

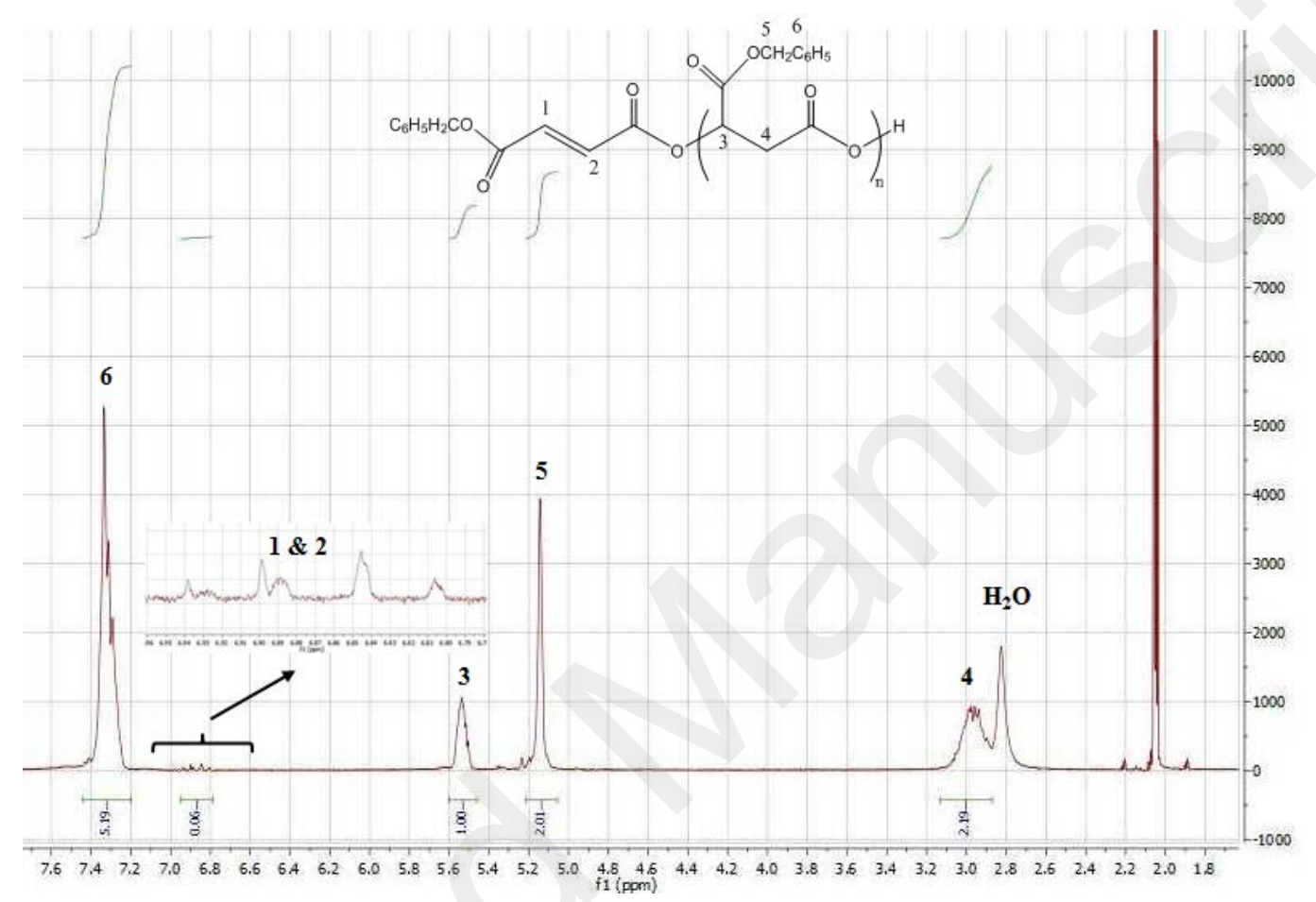

Figure 3. Proton NMR spectrum $\left(\mathrm{CD}_{3} \mathrm{COCD}_{3}, 400 \mathrm{MHz}\right)$ of the purified PMLABe obtained by PPL-catalyzed ROP of MLABe in Tris-HCl buffer $\mathrm{pH} 7$ at $60^{\circ} \mathrm{C}$ under 390 rpm stirring speed during $72 \mathrm{~h}$.

The expansion of the proton NMR spectrum between 6.8 and $7.0 \mathrm{ppm}$ has highlighted the presence of peaks corresponding to ethylenic protons, thus seeming to confirm the hypothetic mechanism described in Figure 2A. Moreover, molar masses of PMLABe calculated using the relative integrations of these ethylenic protons and of the macromolecular chain protons (Figure 3) agree with those measured by SEC in THF ( $\mathrm{M}_{\mathrm{NMR}}=6,800 \mathrm{~g} / \mathrm{mol} ; \mathrm{M}_{\mathrm{w}}=7,570 \mathrm{~g} / \mathrm{mol} ; Ð=1.12$ ). Therefore, these results tend to demonstrate the validity of the proposed lipase-catalyzed ROP mechanism of MLABe involving the abstraction of one of the two "acidic" proton of the lactone's ring probably by the catalytic histidine without the intervention of the catalytic serine.

In order to demonstrate that the lipase-catalyzed ROP of malolactonates does not involve the catalytic serine, we realized the enzymatic ROP of MLABe in presence of a serine knock-out (serine-KO) enzyme. However, PPL mutants being very difficult to obtain, we have selected an easy modifiable recombinant model, the CpLip2 enzyme. Using site-directed mutagenesis of 
serine S180 into an alanine residue, we obtained the serine KO mutant CpLip2_S180A. This mutant was confirmed to show no detectable lipase activity.

If our hypothesis is valid then the CpLip2-Serine KO should lead to the formation of a polymer. Therefore, the polymerizations of MLABe in the presence of CpLip2 and CpLip2_S180A in Tris$\mathrm{HCl}$ buffer $\mathrm{pH} 7$ (optimized conditions for PPL) have been conducted.

As shown by results gathered in Table 3 and Figure 4, PMLABe were obtained in both cases, with, in addition, the presence of the peaks corresponding to ethylenic protons formed by the abstraction of one of the two "acidic” protons of the lactone's ring as shown in Figure 2A.

Table 3. Characteristics of the PMLABe obtained by ROP of MLABe in presence of CpLip2 and CpLip2_S180A enzyme in Tris-HCl buffer pH7 at $60^{\circ} \mathrm{C}$ under $390 \mathrm{rpm}$ stirring during $72 \mathrm{~h}$.

\begin{tabular}{|l|c|c|c|}
\cline { 2 - 4 } \multicolumn{1}{c|}{} & $\mathrm{M}_{\mathrm{RMN}}(\mathrm{g} / \mathrm{mol})$ & $\mathrm{M}_{\mathrm{w}}(\mathrm{g} / \mathrm{mol})^{\mathrm{a}}$ & $\mathrm{Ð}^{\mathrm{a}}$ \\
\hline MLABe + CpLip2 + Tris-HCl buffer pH7 & 4,940 & 3,640 & 1.42 \\
\hline MLABe + CpLip2_S180A + Tris-HCl buffer pH7 & 5,770 & 3,560 & 1.42 \\
\hline
\end{tabular}

a. Measured by SEC in THF at $40^{\circ} \mathrm{C}$, Standards Polystyrenes, Flow Rate $=1 \mathrm{~mL} / \mathrm{min}$



Figure 4. Proton NMR spectrum $\left(\mathrm{CD}_{3} \mathrm{COCD}_{3}, 400 \mathrm{MHz}\right)$ of purified PMLABe obtained: A. by CpLip2-catalyzed ROP of MLABe in Tris-HCl buffer pH7; and B. CpLip2_S180A-catalyzed ROP of MLABe in Tris-HCl buffer $\mathrm{pH}$.

Finally, the CpLip2 has been inactivated by heating at $100^{\circ} \mathrm{C}$ and enzymatic polymerization of MLABe has been conducted with this inactivated enzyme: the ${ }^{1} \mathrm{H}$ NMR spectrum of the crude product has highlighted a monomer conversion of less than $7 \%$ and the absence of polymer. Such results showed that the catalytic histidine certainly plays an important role in the polymerization of malolactonates.

To support further these experimental results, in silico calculations were performed: Figure 5 shows the optimized geometry of S-MLABe and R-MLABe and of the active site of CpLip2 obtained after molecular docking, molecular dynamics equilibration, energy minimization by DFTB and DFT quantum chemical calculations as described in the Computational methods 
section. ETS-NOCV analysis (extended transition state energy decomposition analysis combined with the theory of natural orbitals for chemical valence) ${ }^{33}$ by DFT calculations (BP86D4(EEQ)/DZ level of theory) indicated that the main orbital interaction energy contribution between MLABe and the active site residues was associated to a strong hydrogen bond (-29 kJ/mol and $-23 \mathrm{~kJ} / \mathrm{mol}$ with S- and R-MLABe, respectively) between the $\varepsilon \mathrm{N}$ atom of histidine 365 and one of the "acidic" protons of MLABe (Figure 5).
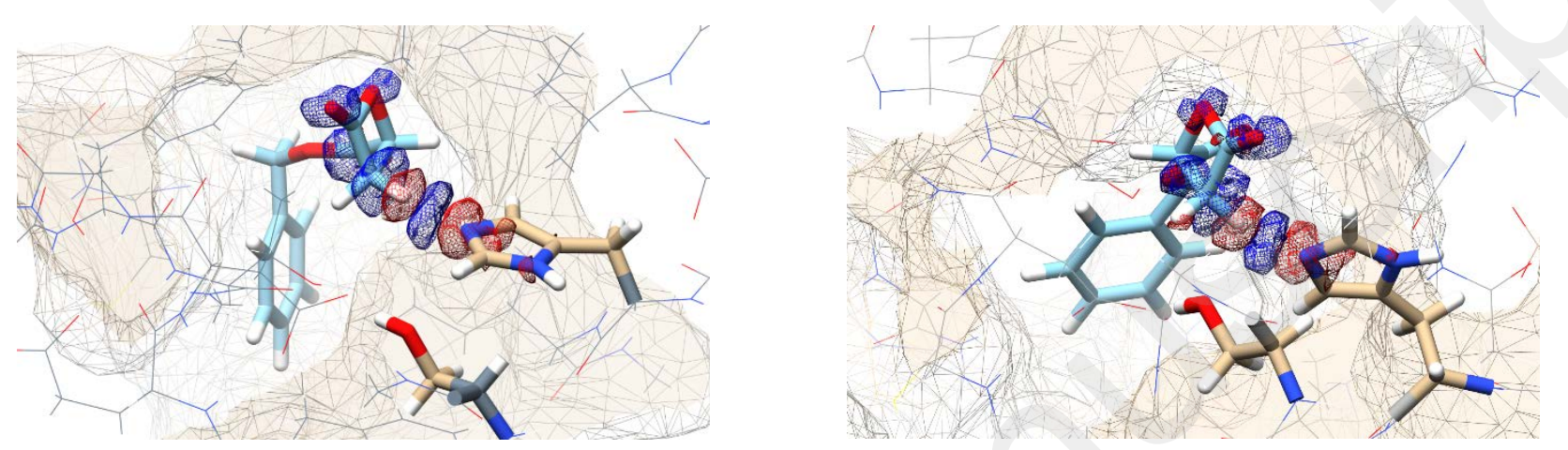

Figure 5. Optimized geometry of the active site of CpLip2 in complex with S-MLABe (left) and R-MLABe (right). The two amino acid residues evidenced are the catalytic serine (S180) and histidine (H365). Interaction energies between the ligand and the active site residues were calculated by DFT (BP86-D4(EEQ)/DZ with water as implicit solvent) according to the ETSNOCV theory. The colored meshes show the deformation of the electron density (depletion in red and accumulation in blue; cut-off 0.007 a.u.) associated to the main NOCV, corresponding to a hydrogen bond between $\mathrm{H} 365$ and a proton of MLABe $(-29 \mathrm{~kJ} / \mathrm{mol}$ with S-MLABe and $-23 \mathrm{~kJ} / \mathrm{mol}$ with R-MLABe).

This result corroborates the hypothesis of a preferred interaction between MLABe and the catalytic histidine instead of the catalytic serine 180, and supports the proposed mechanism of polymerization of MLABe involving histidine, thanks to the presence of "acidic" protons (Figure 2A).

Finally, we tried to polymerize the diMeMLABe in the presence of CpLip2, CpLip2_S180A and imidazole in a catalytic amount in Tris $\mathrm{HCl}$ buffer $\mathrm{pH}$. In all these cases, we did not observe the formation of the corresponding PdiMeMLABe, and unpolymerized and undegraded diMeMLABe was recovered (Figures SI.2C and SI.2D). It is to be noted that molecular docking simulations with (R) and (S)-diMeMLABe and the active site of CpLip2 showed that the steric hindrance brought by the two methyl groups of the lactone ring should prevent the substrate from getting close enough to the catalytic residues (Figure 6). Nevertheless, the impossibility of polymerizing diMeMLABe in the presence of imidazole as initiator, i.e. without the steric hinderance problem encountered with lipases, demonstrates that the absence of "acidic" protons prevents the enzymatic polymerization of these lactones. 

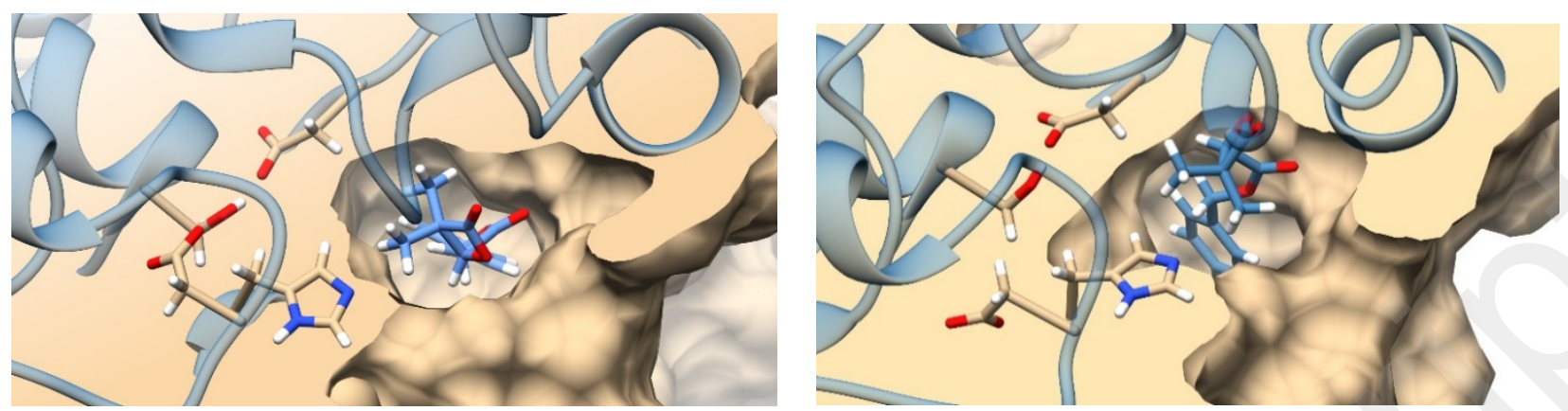

Figure 6. Geometry of the catalytic pocket of CpLip2 in complex with S-diMeMLABe (left) and R-diMeMLABe (right) obtained by molecular docking and energy minimization by molecular dynamics of the whole CpLip2 structure in explicit water. The amino acid residues displayed correspond to the catalytic triad (S180, H365 and D332) and to the D90 residue interacting with the catalytic serine in CpLip2.

All these observations are thus in favor of the proposed mechanism, namely the abstraction of one of the two "acidic" protons of the lactone's ring by the catalytic histidine leading to the formation of a monobenzyl fumarate which then initiates the polymerization of the remaining monomers.

CONCLUSIONS. The obtained results lead us to conclude that the lipase-catalyzed polymerization of MLABe does not follow the canonical mechanism, but results from the abstraction of one of the two "acidic" protons of the lactone's ring by the histidine of the catalytic triad leading to the formation of an activated monomer, i.e. monobenzyl fumarate (Figure 2B), responsible for the polymerization of MLABe. In other words, the lipase acts only as a catalyst to form an activated monomer. However, it would also be responsible for particular conformation adopted by PMLABe chains, thus inducing the presence of crystalline zones even when racemic MLABe are polymerized, conclusion confirmed by the observed melting temperatures measured for PMLABe obtained by enzymatic polymerization of racemic MLABe [unpublished results]. However, additional studies (X-rays, for example) still need to be carried out to elucidate the organization of macromolecular chains induced by the presence of the lipase.

\section{AUTHOR INFORMATION}

Corresponding Author

* sandrine.marion.1@ensc-rennes.fr

\section{Present Addresses}

† Université Clermont Auvergne, CNRS, SIGMA Clermont, ICCF (Institut de Chimie de Clermont-Ferrand) - UMR 6296, 63000 Clermont-Ferrand, France

\section{Author Contributions}

The manuscript was written through contributions of all authors. All authors have given approval to the final version of the manuscript. 


\title{
Funding Sources
}

This work was funded by the « Ecole Nationale Supérieure de Chimie de Rennes » (ENSCR, France), the Centre National de la Recherche Scientifique (CNRS).

\section{ACKNOWLEDGMENT}

The authors gratefully thank the "Ministère de l'Enseignement Supérieur et de la Recherche" (MESR) for financial support of this research (Ph.D grant to H.C.)

\author{
ABBREVIATIONS \\ diMeMLABe: benzyl dimethylmalolactonate \\ MLABe: benzyl malolactonate \\ PdiMeMLABe: poly(benzyl dimethylmalate) \\ PMLABe: poly(benzyl malate) \\ ROP: Ring Opening Polymerization
}

\section{SUPPORTING INFORMATION}

SEC chromatograms of PMLABe obtained under different experimental conditions (Table 2 and Table 3), ${ }^{1} \mathrm{H}$ NMR spectra of pure diMeMLABe and after reaction under various conditions.

\section{REFERENCES}

1. Vert, M. Aliphatic Polyesters: Great degradable polymers that cannot do everything. Biomacromolecules 2005, 6(2), 538-546. Doi: 10.1021/bm0494702.

2. Allcock, H.R.; Lampe, F.W. Polymerization of Cyclic Organic Compounds. In Contemporary Polymer Chemistry, 2nd ed.; Prentice Hall, Englewood Cliffs (New Jersey 07632), USA, 1990, pp 122-123.

3. Jérôme, C.; Lecomte, P. Recent Advances in the Synthesis of Aliphatic Polyesters by RingOpening Polymerization. Adv. Drug Deliv. Rev. 2008, 60(9), 1056-1076. Doi: 10.1016/j.addr.2008.02.008.

4. Tanzi, M.C.; Verderio, P.; Lampugnani, M.G.; Resnati, M.; Dejana, E.; Sturani, E. Cytotoxicity of Some Catalysts Commonly Used in the Synthesis of Copolymers for Biomedical Use. J. Mater. Sci. Mater. Med. 1994, 5(6-7), 393-396. Doi: 10.1007/BF00058971.

5. Shoda, S.; Uyama, H.; Kadokawa, J.; Kimura, S.; Kobayashi, S. Enzymes as Green Catalysts for Precision Macromolecular Synthesis. Chem. Rev. 2016, 116(4), 2307-2413. Doi: 10.1021/acs.chemrev.5b00472.

6. Uyama, H.; Takeya, K.; Kobayashi, S. Synthesis of Polyesters by Enzymatic Ring-Opening Copolymerization Using Lipase Catalyst. Proc. Jpn. Acad. Ser. B 1993, 69(8), 203-207. Doi: 10.2183/pjab.69.203.

7. Knani, D.; Gutman, A.L.; Kohn, D.H. Enzymatic Polyesterification in Organic Media. EnzymeCatalyzed Synthesis of Linear Polyesters. I. Condensation Polymerization of Linear 
Hydroxyesters. II. Ring-Opening Polymerization of $\epsilon$-Caprolactone. J. Polym. Sci. Part Polym. Chem. 1993, 31(5), 1221-1232. Doi: 10.1002/pola.1993.080310518.

8. Kobayashi, S. Enzymatic Ring-Opening Polymerization and Polycondensation for the Green Synthesis of Polyesters. Polym. Adv. Technol. 2015, 26(7), 677-686. Doi: 10.1002/pat.3564.

9. Champagne, E.; Strandman, S.; Zhu, X.X. Recent Developments and Optimization of LipaseCatalyzed Lactone Formation and Ring-Opening Polymerization. Macromol. Rapid Commun. 2016, 37, 1986-2004. Doi: 10.1002/marc.201600494.

10. Casajus, H.; Tranchimand, S.; Wolbert, D.; Nugier-Chauvin, C.; Cammas-Marion, S. Optimization of Lipase-Catalyzed Polymerization of Benzyl Malolactonate through a Design of Experiment Approach. J. Appl. Polym. Sci. 2017, 134(11), 44604 (1-9). Doi: 10.1002/app.44604. 11. Casajus, H.; Saba, S.; Vlach, M.; Vène, E.; Ribault, C.; Tranchimand, S.; Nugier-Chauvin, C.; Dubreucq, E.; Loyer, P.; Cammas-Marion, S.; Lepareur, N. Cell Uptake and Biocompatibility of Nanoparticles Prepared from Poly(benzyl malate) (Co)polymers Obtained through Chemical and Enzymatic Polymerization in Human HepaRG Cells and Primary Macrophages. Polymers, 2018, 10, 1244 (1-19). Doi: 10.3390/polym10111244.

12. Johnson, P.M.; Kundu, S.; Beers, K.L. Modeling Enzymatic Kinetic Pathways for RingOpening Lactone Polymerization. Biomacromolecules 2011, 12(9), 3337-3343. Doi: 10.1021/bm2009312.

13. Matsumura, S. In Enzyme-Catalyzed Synthesis of Polymers; Kobayashi, S., Ritter, H., Kaplan, D., Eds.; Springer Berlin Heidelberg: Berlin, Heidelberg, 2005; Vol. 194, Enzymatic Synthesis of Polyesters via Ring-Opening Polymerization. pp 95-132. Doi: 10.1007/12_030

14. Uyama, H.; Kobayashi, S. Enzymatic Ring-Opening Polymerization of Lactones Catalyzed by Lipase. Chem. Lett. 1993, 22(7), 1149-1150. Doi: 10.1246/cl.1993.1149.

15. Henderson, L.A.; Svirkin, Y.Y.; Gross, R.A.; Kaplan, D.L.; Swift, G. Enzyme-Catalyzed Polymerizations of $\varepsilon$-Caprolactone: Effects of Initiator on Product Structure, Propagation Kinetics, and Mechanism. Macromolecules 1996, 29(24), 7759-7766. Doi: 10.1021/ma960821h. 16. MacDonald, R.T.; Pulapura, S.K.; Svirkin, Y.Y.; Gross, R.A.; Kaplan, D.L.; Akkara, J.; Swift, G.; Wolk, S. Enzyme-Catalyzed Epsilon-Caprolactone Ring-Opening Polymerization. Macromolecules 1995, 28(1), 73-78. Doi: 10.1021/ma00105a008.

17. Svirkin, Y.Y.; Xu, J.; Gross, R.A.; Kaplan, D.L.; Swift, G. Enzyme-Catalyzed Stereoelective Ring-Opening Polymerization of $\alpha$-Methyl- $\beta$-Propiolactone. Macromolecules 1996, 29(13), 4591-4597. Doi: 10.1021/ma951868q.

18. Johnson, P.M.; Kundu, S.; Beers, K.L. Modeling Enzymatic Kinetic Pathways for RingOpening Lactone Polymerization. Biomacromolecules 2011, 12(9), 3337-3343. Doi: 10.1021/bm2009312.

19. Subileau, E.D.; Jan, A.H.; Dubreucq, E. (2018). Lipases/acyltransferases for lipid modification in aqueous media. in: Lipid Modification by Enzymes and Engineered Microbes. Bornscheuer U.T., Elsevier and AOCS Press. 
20. Cammas, S.; Renard, I.; Langlois, V.; Guerin, P. Poly( $\beta$-malic acid: obtaining high molecular weights by improvement of the synthesis route. Polymer 1996, 37(18), 4215-4420. Doi: 10.1016/0032-3861(96)00204-2

21. Brunel, L.; Neugnot, V.; Landucci, L.; Boze, H.; Moulin, G.; Bigey, F.; Dubreucq, E. Highlevel expression of Candida parapsilosis lipase/acyltransferase in Pichia pastoris. J. Biotechnol. 2004, 111, 41-50. Doi: 10.1016/j.jbiotec.2004.03.007

22. Neang, P.; Subileau, M.; Perrier, V.; Dubreucq, E. Homologous yeast lipases/acyltransferases exhibit remarkable cold-active properties. Appl. Microbiol. Biotechnol. 2014, 98, 8927-8936. Doi: 10.1007/s00253-014-5776-6

23. Coulembier, O.; Josse, T.; Guillerm, B.; Gerbaux, P.; Dubois, P. An Imidazole-Based Organocatalyst Designed for Bulk Polymerization of Lactide Isomers: Inspiration from Nature. Chem. Commun. 2012, 48(95), 11695-11697. Doi: 10.1039/c2cc37061a.

24. Subileau, M.; Jan, A.H.; Nozac'h, H.; Perez Gordo, M.; Perrier, V.; Dubreucq, E. The 3D model of the lipase/acyltransferase from Candida parapsilosis, a tool for the elucidation of structural determinants in CAL-A lipases superfamily. Biochimica et Biophysica Acta - Proteins and Proteomics. 2015, 1854, 1400-1411. Doi: 10.1016/j.bbapap.

25. Jan, A.H.; Subileau, M.; Dubreucq E. Characterization and reshaping of a large and hydrophobic nucleophile pocket in lipases/acyltransferases. ChemBioChem. 2018, 19, 1839-1844. 26. Jan, A.H.; Subileau, M.; Deyrieux, C.; Perrier, V.; Dubreucq, E. Elucidation of a key position for acyltransfer activity in Candida parapsilosis lipase/acyltransferase (CpLIP2) and in Pseudozyma antarctica lipase A (CAL-A) by rational design. Biochim. Biophys. Acta - Prot. Proteom. 2016, 1864, 187-194.

27. Nguyen, T.N.; Dubreucq, E.; Perrier, V.; Tran, Q.H.; Charpentier, C.; Charnay, C.; Terki, F.; Jay-Allemand, C.; Bidel, L.P.R. Interactions between trans-resveratrol and CpLIP2 lipase/acyltransferase evidenced by fluorescence and in silico. Food Chem. 2020, 318, 126482, Doi: 10.1016/j.foodchem.2020.126482.

28. Grimme, S.; Bannwarth, C.; Shushkov, P. A robust and accurate tight-binding quantum chemical method for structures, vibrational frequencies, and noncovalent interactions of large molecular systems parametrized for all spd-block elements $(\mathrm{Z}=1-86)$. J. Chem. Theory Comput. 2017, 13, 1989-2009. Doi: 10.1021/acs.jctc.7b00118

29. Klamt, A.; Schuurmann, G. COSMO: A new approach to dielectric screening in solvents with explicit expressions for the screening energy and its gradient. J. Chem. Soc. Perkin Trans. 1993, 2, 799-805. Doi:10.1039/P29930000799.

30. Trott, O.; Olsonn A.J.; AutoDock Vina: improving the speed and accuracy of docking with a new scoring function, efficient optimization and multithreading. J. Comput. Chem. 2011, 31, 455461. Doi:10.1002/jcc.21334.

31. Pettersen, E.F.; Goddardn T.D.; Huangn C.C.; Couch, G.S.; Greenblatt, D.M.; Meng, E.C.; Ferrin, T.E. UCSF Chimera - A visualization system for exploratory research and analysis. $J$. Comput. Chem. 2004, 25, 1605-1612. Doi:10.1002/jcc.20084. 
32. Caldeweyher, E.; Ehlert, S.; Hansen A.; Neugebauer H.; Spicher S.; Bannwarth C.; Grimme, S. A generally applicable atomic-charge dependent London dispersion correction J. Chem. Phys. 2019, 150, 154122. Doi: 10.1063/1.5090222.

33. Mitoraj, M.; Michalak, A. Natural orbitals for chemical valence as descriptors of chemical bonding in transition metal complexes. J. Mol. Model. 2007, 13, 347-355. Doi: 10.1007/s00894006-0149-4.

34. Casas-Godoy, L.; Duquesne, S.; Bordes, F.; Sandoval, G.; Marty, A. Lipases: An Overview. Methods Mol. Biol. Clifton NJ 2012, 861, 3-30. Doi: 10.1007/978-1-61779-600-5_1.

35. Rauwerdink, A.; Kazlauskas, R.J. How the Same Core Catalytic Machinery Catalyzes 17

Different Reactions: The Serine-Histidine-Aspartate Catalytic Triad of $\alpha / \beta$-Hydrolase Fold Enzymes. ACS Catal. 2015, 5(10), 6153-6176. Doi: 10.1021/acscatal.5b01539.

36. Li, C.; Feng, X.W.; Wang, N.; Zhou, Y.J.; Yu, X.Q. Biocatalytic Promiscuity: The First LipaseCatalysed Asymmetric Aldol Reaction. Green Chem. 2008, 10(6), 616-618. Doi: 10.1039/B803406K.

37. Albertsson, A.C.; Varma, I.K. Recent Developments in Ring Opening Polymerization of Lactones for Biomedical Applications. Biomacromolecules 2003, 4(6), 1466-1486. Doi: 10.1021/bm034247a.

38. Kricheldorf, H.R.; Scharnagl, N.; Jedlinski, Z. Polylactones 33. The Role of Deprotonation in the Anionic Polymerization of $\beta$-Propiolactone. Polymer 1996, 37(8), 1405-1411. Doi: 10.1016/0032-3861(96)81139-6

39. Hofman, A.; Słomkowski, S.; Penczek, S. Structure of Active Centers and Mechanism of the Anionic Polymerization of Lactones. Makromol. Chem. 1984, 185(1), 91-101. doi: 10.1002/macp.1984.021850110.

40. Mabille, C.; Masure, M.; Hémery, P.; Guérin, Ph. Obvious complexity of the anionic polymerization of malolactonic acid esters. Polym. Bull. 1998, 40, 381-387. 


\section{For Table of Content Only - Lipase catalyzed ring- opening polymerization of benzyl malolactonate: an unusual mechanism?}

Hubert Casajus, Eric Dubreucq, Sylvain Tranchimand, Véronique Perrier, Caroline NugierChauvin, Sandrine Cammas-Marion

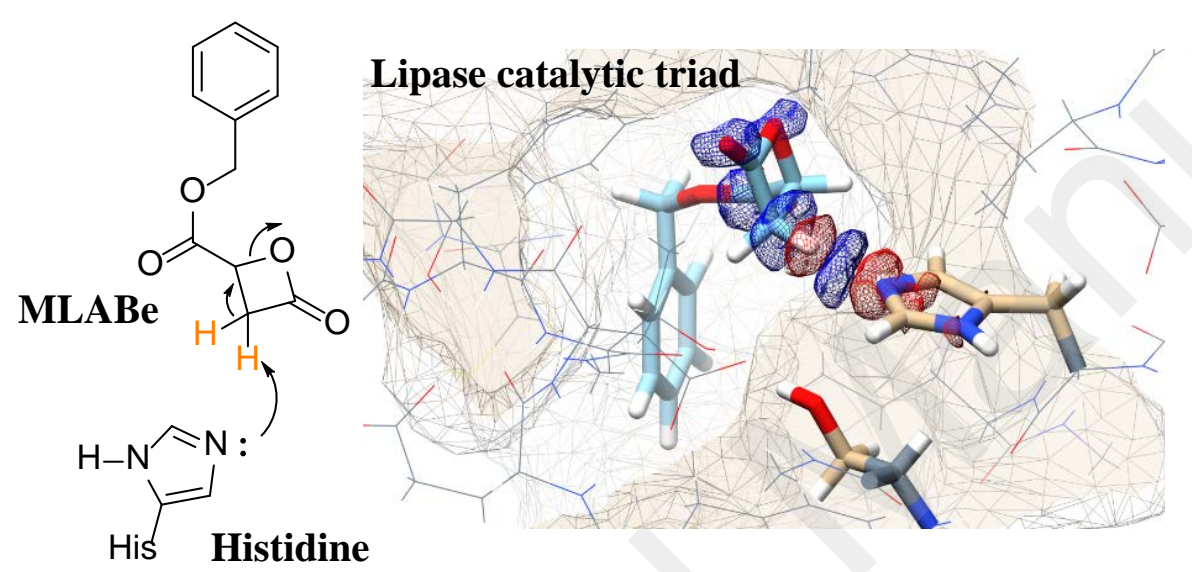<smiles>CC(O)(O)CC(CC(=O)/C=C/C(=O)OCc1ccccc1)OCc1ccccc1</smiles>

Enzymatic PMLABe 